\title{
The risks of overdiagnosis
}

\section{Screening mammograms catch some cancers that pose little threat. Alexandra Barratt explains why she may decide to skip the scans.}

$\mathrm{I}$ magine a busy breast cancer clinic in a place such as Washington DC, London or my home city of Sydney, where women regularly have screening mammograms. In the waiting room sit women in their 40s, 50s and older who have breast cancer that was found by screening. They are scared and deeply uncertain about their future. But do they all need to be there? No, because some of them have been 'overdiagnosed ${ }^{1-3}$ - they are about to have treatment for a breast cancer that would not have caused any health problems had it been left undetected and untreated.

In an ideal scenario, breast-cancer screening would find potentially lethal breast cancers before they have caused symptoms (such as a lump). Finding these cancers early would permit the best possible treatment and mean fewer breast cancer deaths ${ }^{2}$. This was the premise that underpinned screening programmes, but, increasingly, research shows that the picture is much more complicated.

Breast cancer takes many forms - some indolent and harmless, and some very aggressive and lethal that grow and spread rapidly. Because a screening mammogram is a snapshot in time, it is more likely to catch a slow-growing cancer than a fast-growing one ${ }^{3}$. In other words, it leads to overdiagnosis because of its tendency to detect the cancers that are unlikely to be harmful.

As a woman in my 50s, this is not just an academic issue. I have two options: I can have regular screening (every 2 years from age 50 to 74 is the recommended schedule in Australia), or I can choose not to. To decide, I need to consider the potential outcomes. One possibility is that my mammograms will always be normal and that will be that. Or perhaps one of my mammograms will not be normal and I will have further tests, which will show I do not have cancer. This is called a false positive. If I regularly go for screening for 20 years or so, there is more than a $40 \%$ chance that I will have one of these scares $^{4,5}$. I would feel anxious, and it might take me some time to recover, but, on its own, I do not think the threat of a false alarm would stop me from being screened. The third possibility is that I am diagnosed with breast cancer. Like the women in the waiting room, I would be advised to have surgery (to remove the cancer or the entire breast), and probably radiotherapy, along with hormone therapy for 5 or more years.

So why would I even consider not getting screened? Am I not worried about dying of breast cancer? Yes, of course I am. But I'm also worried by the possibility that I could be seriously harmed by the treatment of a cancer that would never have affected my health.

Radiotherapy will increase my risk of heart disease, especially if the breast cancer is on the left, the same side as the heart. Cardiovascular disease killed my mother and three of my grandparents, so that is a big concern for me. I would almost certainly experience some of the common side effects of hormone therapy, such as tamoxifen, including mood problems, low libido and vaginal dryness - and an increased risk of blood clots and stroke. Both my daughters would feel anxious and would, forever after, have a 'family history of breast cancer'. Importantly to me, the psychological impact and physical risks of being treated for an overdiagnosed cancer would start straightaway. As I get older, I find that having good health and enjoying my life today becomes more and more important than the future.

At present, scientists and doctors cannot identify an 'overdiagnosed' cancer at the individual level because we do not have tests that can reliably distinguish between breast cancers that will progress to cause health problems and those that will not. Overdiagnosis can only be inferred from population-level statistics, so I could never be certain whether I had been overdiagnosed or not. I'm not sure that is a Pandora's box that I'm willing to open. This is the conundrum at the heart of overdiagnosis, and the only way I can avoid it is by not getting screened in the first place.

I still might, however, decide to get screened because of the small chance that it could prevent me dying from breast cancer. By some estimates, screening 1,000 women regularly for 20 years might prevent 4 breast cancer deaths ${ }^{6}$. Some estimates are even lower ${ }^{4,7}$. That means that there is roughly $0.4 \%$ chance that screening will make the difference between me dying of breast cancer and not. Estimates vary, but I'm more likely to be overdiagnosed than to have my life saved - three to ten times more likely ${ }^{6,7}$.

To be clear, if I decide not to have screening, that does not mean I'm pretending breast cancer does not exist. If I notice a change in my breasts such as a lump, I will see my doctor without delay to have it tested. And if, in the future, the science about breast-cancer screening changes, I will carefully consider the new information. For now, I will do what I can to minimize my risk of breast cancer by watching my weight, incorporating exercise into my life and moderating my alcohol intake.

Whatever I decide, my decision should not determine that of anyone else. Women have different bodies, families, circumstances, preferences and fears. What matters is that each woman has access to the best information that science can provide to allow her to choose wisely what is best for her.

Alexandra Barratt is an epidemiologist at the University of Sydney, Australia. e-mail Alexandra.Barratt@sydney.edu.au

1. Carter, J. L., Coletti, R. J. \& Harris, R. P. Br. Med. J. 350, g7773 (2015).

2. Esserman, L., Shieh, Y. \& Thompson, I. J. Am. Med. Assoc. 302, 1685-1692 (2009).

3. Welch, H. G. \& Black, W. C. J. Natl Cancer Inst. 102, 605-613 (2010).

4. Welch, H. G. \& Passow, H. J. JAMA Intern. Med. 174, 448-454 (2014).

5. Hersch, J. et al. Lancet 385, 1642-1652 (2015).

6. Marmot, M. G. et al. Lancet 380, 1778-1786 (2012).

7. Gøtzsche, P. C. \& Jørgensen, K. J. Cochrane Database Syst. Rev. 2013, CD001877 (2013) 